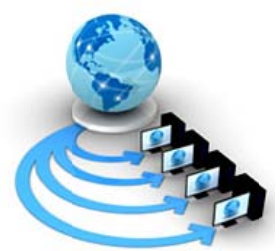

Volume 9, No. 1, January-February 2018

International Journal of Advanced Research in Computer Science

REVIEW ARTICLE

\author{
Available Online at www.ijarcs.info
}

\title{
IMAGE \& VIDEO COMPRESSION WITH RATE DISTORTION APPROACH: A REVIEW
}

\author{
Preeti \\ M.Tech Scholar, \\ ECE Department, D.C.R.U.S.T,Murthal
}

\author{
Gitanjali Pandove \\ Associate Professor, \\ ECE Department, D.C.R.U.S.T, Murthal
}

\begin{abstract}
In this paper, a rate distortion approach is studied to solve the optimization purpose. Now a day, digital video compression technique is most popular for cost reduction. Many applications such as digitized music or internet broadcasting the movie, provided by the digital video industry cannot be forgotten. These attributes of digital video provides continue progression in compression technology \& improvement on different media storage or audio / video service streaming. A RDO(Rate distortion optimization) technique is an optimization technique which involves diamond and exhaustive search. An exhaustive search process is involved to determine the optimal quantized transform coefficient, used in block code. The computational cost of exhaustive search quantization is more expensive than conventional quantization.
\end{abstract}

Keywords:Rate distortion approach, High definition television, Spatial smoothness map, Support vector machine, Rate distortion optimization, DCT, Quantization parameter.

\section{INTRODUCTION}

A conventional system setting for researching on video compression is the pair of encoder and decoder, assuming abundant computation power for encoding, limited computation power for decoding, and no diversity for spatial and temporal resolutions. Under this circumstance, a critical question is what the best rate distortion (RD) trade-off is, which is the first problem to be tackled [1]. Furthermore, if we consider the spatial resolution diversity between the capturing unit and the displaying unit, there is a transcoding problem, which involves converting the spatial resolution for a compressed source. This transcoding task with spatial resolution conversion motivates the second major work in this thesis for image/video down-sampling in the discrete cosine transformation (DCT) domain [2]. Lossy video compression under the conventional system setting with abundant encoding power generally adopts a hybrid structure, where several different compression techniques such as motion prediction, transform, quantization, and entropy coding are employed together. In general, this is referred to as hybrid video compression. This structure follows an intuitive understanding of video data about the temporal redundancy (similarity between Video compression in a practical multimedia system may be customized by different system settings such as its device diversities and the data delivery method [3] Conventional hybrid video compression assumes only the pair of encoder and decoder, overlooking the device diversities and the data delivery method. Transcoding considers the spatial resolution diversity, and/or the temporal resolution diversity, and/or channel bandwidth diversity through a network. Distributed video coding addressesthecomputationpowerdiversity, technically speaking[4].

The rest of research paper is design as follows. The overall previous work is described in Section II. Section
IIIdescribes problem formulation. Performance parameter describe in section IV. Finally, Section V describes the conclusion of paper.

\section{LITERATURE REVIEW}

This section will provide the brief description and highlights the contribution, remarks and factors of the work done by the researchers. Many attempts have been made in the past to achieve the maximum peak signal to noise ratio.

Qin Huang et.al described about RBF technique.Linear Kernel and Non-linearKernel technique was used. For linear kernel technique GM,SSM and STSM was 2.392 .49 and 2.43 respectively. For non-linear kernel techniques GM,SSM and STSM were2.55,2.38and 1.88 respectively [1].

Takashi Tanaka et.al discussed about SRD Approach for Gaussian SRD problem, Linear Gaussian sensor design problem, SRD optimization as max-det problem and Maxdet problem as SDP respectively. The result was found that it provides min. downlink bandwidth for satellite attitude determination[2].

AlexandreMercat et.al described about Quad-tree partitioningIntra encoding and RD-cost technique.The best results were obtained for the video sequences of class $\mathrm{F}$ with up to $36 \%$ BD-BR savings between CDC and the inverse allocator [3].

MohammadrezaStephane et.al described about Promising Rate distortion approach and non -promising modes of Rate distortion approach. The major findings from his research were encoding time reduction,BD PSNR,BD Rate were 41.8 $\%, 0.058 \mathrm{db}$ and $1.24 \%$ respectively [4].

YanboGeo et.al described about a layer-based temporal dependent RDO method for RA-HVC and Embedded 
Temporal propagation Chain for Random- Access Hierarchical Video Coding. The average BD-rate gain,BDrate saving was $1.4 \%$ and $3.8 \%$ respectively. For the proposed method, theaverage ETR of each class was about $103 \% \sim 105 \%$, and the average ETR of all sequences is about $104 \%[5]$.

Shuichi Ohno et.al describedAboutquantizewith an error feedback filter and design of the Noise Shaping Filters. The result was found that MSEs of the optimal feedback quantize,the optimal feedback quantize is-10, -20, -30 dbrespectively[6].
SichuanGoo et.al described about Syntax-based contextadaptive Binary Arithmetic Coding the Gloom coding, which was widely used in HEVC. The PSNR loss, Rate computation reduction were 0.0428 and $28.4 \%$ respectively [7].

Yi Liu et.al described about Locally Adaptive Resolution, Quad tree Partitioning, Quantization Process and Proposed RDO Model. The result was found that Gradient Entropy and Bit Rate for Lossless Coding was 5.892 and 12.460 respectively

Table1. Literature Review Table

\begin{tabular}{|c|c|c|c|c|}
\hline Authors & Paper Title & $\begin{array}{l}\text { Research Methodology } \\
\text { used }\end{array}$ & Major Findings & $\begin{array}{l}\text { Research } \\
\text { prospects }\end{array}$ \\
\hline $\begin{array}{l}\text { Qin Huang, Haitian } \\
\text { Wang, Sung Chang } \\
\text { Lim, Hue Yong Kim, }\end{array}$ & $\begin{array}{lr}\text { Measure } & \text { and } \\
\text { Prediction } & \text { of } \\
\text { HEVC } & \\
\text { Perceptually } & \text { Loss } \\
\text { /Lossless } & \\
\text { Boundary } & \text { QP } \\
\text { Values } & \end{array}$ & $\begin{array}{l}\text { Linear Kernel Non-linear } \\
\text { RBF Kernel } \\
\text { JND-based quality } \\
\text { assessment dataset for } \\
\text { HEVC-coded video }\end{array}$ & $\begin{array}{l}\text { Linear Kernel } \\
\text { GM : } 2.39 \\
\text { SSM : } 2.49 \\
\text { STSM }: 2.43 \\
\\
\text { Non Linear } \\
\text { Kernel } \\
\text { GM : } 2.55 \\
\text { SSM : } 2.38 \\
\text { STSM }: 1.88\end{array}$ & $\begin{array}{l}\text { By } \\
\text { characterizing } \\
\text { major artifacts } \\
\text { and deriving } \\
\text { effective } \\
\text { features, the } \\
\text { proposed SVR } \\
\text { based prediction } \\
\text { system can } \\
\text { predict the first } \\
\text { JND value for } \\
\text { each Goop. }\end{array}$ \\
\hline $\begin{array}{l}\text { Takashi Tanaka, } \\
\text { Kwang-Ki K. Kim, } \\
\text { Pablo A. Parrilo, and } \\
\text { Sanjoy K. Mitter }\end{array}$ & $\begin{array}{l}\text { Semi definite } \\
\text { programming } \\
\text { Approach } \\
\begin{array}{l}\text { Gaussian } \\
\text { Sequential }\end{array} \\
\begin{array}{l}\text { Ristortion } \\
\text { Rate- } \\
\text { ofs }\end{array}\end{array}$ & $\begin{array}{l}\text { SRD Approach for } \\
\text { 1. Gaussian SRD problem } \\
\text { 2. Linear Gaussian sensor } \\
\text { design problem } \\
\text { 3. SRD optimization as } \\
\text { max-det problem } \\
\text { 4. Max-det problem as } \\
\text { SDP }\end{array}$ & $\begin{array}{l}\text { It provides min. } \\
\text { downlink bandwidth } \\
\text { for satellite attitude } \\
\text { determination }\end{array}$ & $\begin{array}{l}\text { The implication } \\
\text { is that Gaussian } \\
\text { SRD problems } \\
\text { are efficiently } \\
\text { solvable using } \\
\text { standard SDP } \\
\text { solvers. }\end{array}$ \\
\hline $\begin{array}{l}\text { AlexandreMercat , } \\
\text { Florian Arrestier, } \\
\text { WassimHamidouche, } \\
\text { MaximePelcat, } \\
\text { Daniel Menard }\end{array}$ & $\begin{array}{l}\text { Constrain the } \\
\text { Docile CTUs: an } \\
\text { In-Frame } \\
\text { Complexity } \\
\text { Allocator for } \\
\text { HEVC Intra } \\
\text { Encoders }\end{array}$ & $\begin{array}{l}\text { Quad-tree partitioning } \\
\text { Intra encoding } \\
\text { RD-cost }\end{array}$ & $\begin{array}{l}\text { The best results are } \\
\text { obtained for the video } \\
\text { sequences } \\
\text { of class F with up to } \\
36 \% \text { BD-BR savings } \\
\text { between CDC and } \\
\text { the inverse allocator. }\end{array}$ & $\begin{array}{l}\text { 1.RD-cost is } \\
\text { linked to the } \\
\text { partitioning } \\
\text { depths of CTUs. } \\
\text { 2. CTUs with } \\
\text { low RD-cost } \\
\text { have less } \\
\text { increase of bit } \\
\text { rates } \\
\text { and/or distortion } \\
\text { than CTUs with } \\
\text { high RD-cost } \\
\text { when } \\
\text { constrained }\end{array}$ \\
\hline $\begin{array}{l}\text { Mohammadreza } \\
\text { StephaneCoulombe }\end{array}$ & $\begin{array}{l}\text { RDO Cost } \\
\text { Modeling for Low } \\
\text { Complexity } \\
\text { HEVC } \\
\text { Coding }\end{array}$ & $\begin{array}{l}\text { Promising Rate distortion } \\
\text { approach } \\
\text { Non promising modes of } \\
\text { Rate distortion approach }\end{array}$ & $\begin{array}{l}\text { Encoding } \\
\text { reduction : } 41.8 \% \\
\text { BD PSNR : } 0.058 \mathrm{db} \\
\text { BD Rate : } 1.24 \%\end{array}$ & $\begin{array}{l}\text { RDO Cost } \\
\text { Modeling to } \\
\text { reduce the } \\
\text { computational } \\
\text { complexity of } \\
\text { HEVC intra } \\
\text { coding }\end{array}$ \\
\hline $\begin{array}{l}\text { Yambol Ago, Coe } \\
\text { Zhu, Shay Li and } \\
\text { Tawny Yang }\end{array}$ & $\begin{array}{l}\text { Layer-Based } \\
\text { Temporal } \\
\text { Dependent Rate- }\end{array}$ & $\begin{array}{l}\text { A layer-based temporal } \\
\text { dependent RDO method } \\
\text { for RA-HVC }\end{array}$ & $\begin{array}{l}\text { average } \text { BD-rate gain } \\
: 1.4 \% \\
\text { BD-rate saving : }\end{array}$ & $\begin{array}{l}\text { It is shown that } \\
\text { the } \\
\text { Temporal }\end{array}$ \\
\hline
\end{tabular}




\begin{tabular}{|c|c|c|c|c|}
\hline & $\begin{array}{l}\text { Distortion } \\
\text { Optimization in } \\
\text { Random-Access } \\
\text { Hierarchical Video } \\
\text { Coding }\end{array}$ & \begin{tabular}{lr} 
Embedded & \multicolumn{2}{c}{ Temporal } \\
propagation & Chain for \\
Random- & Access \\
Hierarchical & Video \\
Coding &
\end{tabular} & $\begin{array}{l}3.8 \% \\
\text { For the proposed } \\
\text { method, the } \\
\text { Average ETR of each } \\
\text { class is about } \\
103 \% \text { 105\%, and the } \\
\text { average ETR of all } \\
\text { sequences is about } \\
104 \% \text {. }\end{array}$ & $\begin{array}{l}\text { dependent RDO } \\
\text { can be } \\
\text { formulated as } \\
\text { minimizing the } \\
\text { Aggregated } \\
\text { distortions of the } \\
\text { to-be-coded unit } \\
\text { and its affected } \\
\text { Units subject to a } \\
\text { rate constraint. }\end{array}$ \\
\hline $\begin{array}{l}\text { Shuichi Ohio, } \\
\text { Teriyaki Shitake, M. } \\
\text { Rowan Tariq, and } \\
\text { Masaaki Niagara }\end{array}$ & $\begin{array}{l}\text { Rate-Distortion } \\
\text { Analysis of } \\
\text { Quantizes with } \\
\text { Error Feedback }\end{array}$ & $\begin{array}{l}\text { 1. Quantize with an error } \\
\text { feedback filter } \\
\text { 2. Design of the Noise } \\
\text { Shaping Filters }\end{array}$ & $\begin{array}{l}\text { MSEs of the optimal } \\
\text { feedback quantize , } \\
\text { the optimal feedback } \\
\text { quantize is -10, }-20,- \\
30 \text { db respectively }\end{array}$ & $\begin{array}{l}\text { The amplitude } \\
\text { response of the } \\
\text { optimal error } \\
\text { feedback filter } \\
\text { that minimizes } \\
\text { the MSE can be } \\
\text { parameterized by } \\
\text { one parameter } \\
\text { and can be found } \\
\text { numerically. }\end{array}$ \\
\hline $\begin{array}{l}\text { SanchuanGuo, } \\
\text { Zhenyu Liu, } \\
\text { Dongsheng Wang, } \\
\text { Qingrui Han and } \\
\text { Yang Song }\end{array}$ & $\begin{array}{l}\text { Linear Rate } \\
\text { Estimation Model } \\
\text { for HEVC RDO } \\
\text { Using Binary } \\
\text { Classification } \\
\text { Based Regression }\end{array}$ & $\begin{array}{l}\text { Syntax-based context- } \\
\text { adaptive Binary } \\
\text { Arithmetic Coding the } \\
\text { Golomb coding which is } \\
\text { wildly used in HEVC, we } \\
\text { devise the fast } \\
\text { method to estimate its rate } \\
\text { cost }\end{array}$ & $\begin{array}{l}\text { PSNR loss : } 0.0428 \\
\text { Rate computation } \\
\text { reduction : } 28.4 \%\end{array}$ & $\begin{array}{l}\text { The } \\
\text { classification } \\
\text { based linear } \\
\text { regression } \\
\text { method to derive } \\
\text { the fast } \\
\text { estimation model } \\
\text { of rate } \\
\text { Cost. }\end{array}$ \\
\hline $\begin{array}{lr}\text { Yi Liu, } & \text { Olivier } \\
\text { Defogs, } & \text { François } \\
\text { Pasteur, } & \\
\text { KhouloudSamrouth }\end{array}$ & $\begin{array}{l}\text { Low Complexity } \\
\text { RDO Model for } \\
\text { Locally Subjective } \\
\text { Quality } \\
\text { Enhancement in } \\
\text { LAR Coder }\end{array}$ & $\begin{aligned} \text { Locally } & \text { Adaptive } \\
\text { Resolution } & \\
\text { 1. } & \text { Quad tree } \\
& \text { Partitioning } \\
\text { 2. } & \text { Quantization } \\
& \text { Process } \\
\text { 3. } & \text { Proposed RDO } \\
& \text { Model }\end{aligned}$ & $\begin{array}{l}\text { Gradient Entropy : } \\
5.892 \\
\text { Bit Rate for Lossless } \\
\text { Coding : } 12.460\end{array}$ & $\begin{array}{l}\text { Although } \\
\text { objective quality } \\
\text { is not improved } \\
\text { from the } \\
\text { experimental } \\
\text { results, } \\
\text { subjective the } \\
\text { quality is } \\
\text { enhanced visibly }\end{array}$ \\
\hline
\end{tabular}

\section{PROBLEM FORMULATION}

The quantization is not implemented on residues for lossless model in HEVC and to avoid distortion. As due to this a new method i.e. lambda model is implemented in RateDistortion Optimization (RDO), where lambda is an factor that is based on quantization, which is independent to the lossless coding [9]. This paper firstly shows the role of lambda that it played in RDO of HEVC. It also shows the simulation results that are based on the annealing algorithm. This techniques is proposed to get the most appropriate lambda result for every large coding unit. If we consider the complexity of computer than these methods are not so efficient for this we proposed some prediction to improve RDO process [10].

The main objective of the paper is to study and analyze various rate distortion optimization in HVEC and H.264/AVC to improve the video encoding efficiency propose and design HEVC with variable size of coding unit using Rate-Distortion Optimization (RDO).

\section{PERFORMANCE PARAMETER}

The performance ofimage and video compressionis measure with compression ratio, global PSNR, average PSNR, SSSIM and gradient entropy.

\section{Compression Ratio}

Compression ratio basically an ration between the sizes of files that are before compression process and after compression process respectively. This ration gives an theoretical value that by how much times the files is compressed from original file. For an algorithm the compression ratio must be larger [11].

\section{Compression Factor}

$$
\text { Compression Ratio }=\frac{\text { Size after Compression }}{\text { Size before Compression }}
$$

The inverse of compression ratio is called compression factor or it is an ratio of file before compression and after compression respectively [12].

$$
\text { Compression Factor }=\frac{\text { Size before Compression }}{\text { Size after Compression }}
$$




\section{Compression Time}

For calculation the time take by compression and to decompress the file has to be taken in separate account. Because in some application the decompression time is more important factor while in some other application the combination of both compression and decompression time plays an important role. For an acceptable result the time taken by compression and decompression must be smaller than algorithm [12]. The time taken for compression and decompression is totally depended on the computing devices.

\section{Global PSNR Rate}

MPEG-4Signal-to-noise ratio (often abbreviated SNR or $\mathrm{S} / \mathrm{N}$ )is an measure that are being used in technology field now a day's which gives an comparison between the required signal with the background noise. So in mathematical terms this can be defined as the ration between signal power and noise power. This term is defined in decibels. If the ration is greater than $1: 1$ (greater than $0 \mathrm{~dB}$ ) then this shows that signal is more than the noise. As a SNR is only applicable for the electrical signal but this technique can be employed for any kind of signal and gives satisfactory results. (such as isotope levels in an ice core or biochemical signaling between cells) [13].

\section{Average PSNR Rate Video Samples (PSNR global)}

The difference between "PSNR" and "APSNR" is in the way of average PSNR calculation for a sequence. The requisite way to compute average PSNR for an sequence is to first find the mean square error for all the frames and after this we can calculate the PSNR value by the conventional simple equations of PSNR [14].

\section{SSIM Rate Video Samples (SSIM)}

SSI basically is based on the results of three factors after finding the results of these three factors all the results are combined to give a final result i.e. (luminance similarity, contrast similarity and structural similarity). There are mainly two implanted SSIM present which are fast and precise. The difference between these two implementation is that in Fast SSIM it uses box filter and in precise SSIM it uses gauss blur[15].

\section{CONCLUSION}

In this review paper different video and audio compression techniques with its simulation parameters global PSNR , average PSNR and SSIM are studied. The different techniques are used to improve the performance parameter.These video are used for personal and commercial used.A rate distortion is more efficient than other video compression technique like quad tree partition technique, syntax based arithmetic coding. In context to complete survey it is clear that RDO technique have less time complexity in comparison to RBF kernel technique.

\section{REFERENCES}

[1] Qin Huang et.al "Measure and Prediction of HEVC Perceptually Lossy/Lossless Boundary QP Values”IEEE Data Compression Conference (DCC), pp 42-51, 2017.

[2] Takashi Tanaka et.al "Semi definite programming Approach to Gaussian Sequential Rate-Distortion Tradeoffs"IEEE Transactions on Automatic Control , pp1896-1910 , 2017

[3] AlexandreMercat et.al "Constrain the Docile CTUs: an InFrame Complexity Allocator for HEVC Intra Encoders”IEEE International Conference on Acoustics, Speech and Signal Processing (ICASSP), pp 1163 - 1167, 2017.

[4] Mohammadrezaet.al "RDO Cost Modeling for Low Complexity HEVC Intra Coding” IEEE Canadian Conference on Electrical and Computer Engineering , pp 1-5 , 2016.

[5] YanboGao et.al "Layer-Based Temporal Dependent RateDistortion Optimization in Random-Access Hierarchical Video Coding"IEEE 18th International Workshop on Multimedia Signal Processing, pp 1-6, 2016.

[6] Shuichi Ohno"Rate-Distortion Analysis of Quantizes with Error Feedback” IEEE journal of multimedia communication, Vol 74, issue 4, pp 406-416, Sept 2016.

[7] SanchuanGuo"Linear Rate Estimation Model for HEVC RDO Using Binary Classification Based Regression”IEEE Data Compression Conference, pp 406 -410, 2014.

[8] Yi Liu "Low Complexity RDO Model for Locally Subjective Quality Enhancement in LAR Coder”IEEE International Conference on Signal and Image Processing Applications, pp $176-181,2013$.

[9] B. Li, J.Z. Xu, D. Zhang and H.Q. Li, "QP refinement according to Lagrange multiplier for High Efficiency Video Coding,’IEEE Int. Symp. Circuits Syst. (ISCAS), Beijing, China, May 2013.

[10] F. Bossen, "Common test conditions and software reference configurations,"document JCTVC-L1100, Geneva, Switzerland, Jan 2013

[11] G.J. Sullivan, J.R. Ohm, W.J. Han and T. Wiegand, "Overview of the High Efficiency Video Coding (HEVC) Standard,”IEEE Trans. Circuits Syst. Video Technol., vol. 22, no.12, pp. 1649 - 1668, Dec. 2012.

[12] T. Summers, F. Cortesi, and J. Lygeros, "On sub modularity and controllability in complex dynamical networks,"IEEE Transactions on Control of Network Systems, vol. PP, no. 99, 2015.

[13] A. M. Tourapis, O. C. Au, and M. L. Liou, "Predictive motion vector field adaptive search technique (PMVFAST) Enhancing block based motion estimation,” in Proc. Visual Communications and Image Processing 2001 (VCIP-2001), San Jose, CA, Jan.2001.

[14] K. Cheung and L. M. Po,“A hierarchical block motion estimation algorithm using partial distortion measure," in Proc. International Conference on Image Processing, Apr. 1997, pp. 606-609.

[15] J. B. Xu, L. M. Po, and C. K. Cheung, "Adaptive motion tracking block matching algorithms for videocoding,"IEEE Trans. Circuits and Syst. Video Technol., vol. 97, pp. 10251029,Oct. 1999. 